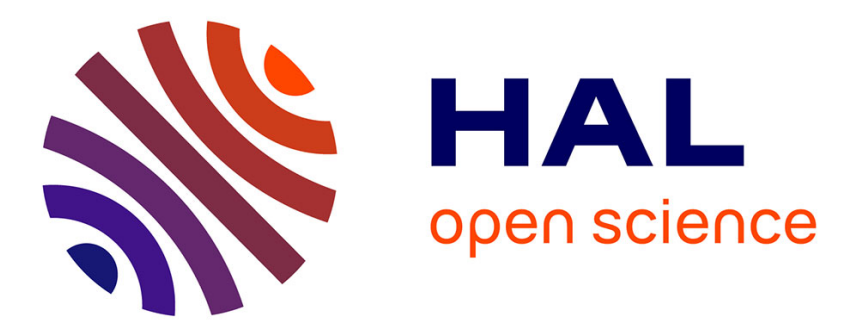

\title{
Constitutive model for flake graphite cast iron automotive brake disks: From macroscopic multiscale models to a 1D rheological description
}

L Augustins, René R Billardon, François Hild

\section{- To cite this version:}

L Augustins, René R Billardon, François Hild. Constitutive model for flake graphite cast iron automotive brake disks: From macroscopic multiscale models to a 1D rheological description. Continuum Mechanics and Thermodynamics, 2016, 28, pp.1009-1025. 10.1007/s00161-015-0448-z • hal-01383890

\author{
HAL Id: hal-01383890 \\ https://hal.science/hal-01383890
}

Submitted on 19 Oct 2016

HAL is a multi-disciplinary open access archive for the deposit and dissemination of scientific research documents, whether they are published or not. The documents may come from teaching and research institutions in France or abroad, or from public or private research centers.
L'archive ouverte pluridisciplinaire HAL, est destinée au dépôt et à la diffusion de documents scientifiques de niveau recherche, publiés ou non, émanant des établissements d'enseignement et de recherche français ou étrangers, des laboratoires publics ou privés. 


\title{
Constitutive model for flake graphite cast iron automotive brake disks
}

\section{From macroscopic multiscale models to a 1D rheological description}

\author{
L. Augustins · R. Billardon · F. Hild
}

Received: date / Accepted: date

\begin{abstract}
One of the critical points of the thermomechanical fatigue design process is the correct description of the cyclic behavior of the material. This work focuses on the material of automotive brake disks, namely, flake graphite cast iron. The specificity of this material is its asymmetric behavior under tensile and compressive loadings, which is due to the shape of graphite that acts as small cracks. Multiscale models inspired from the literature are first presented. They lead to a good description of the material behavior under cyclic loadings. An elasto-viscoplastic constitutive model is then proposed in a one dimensional setting in order to accurately describe cyclic tests from room temperature up to $600^{\circ} \mathrm{C}$.
\end{abstract}

Keywords Flake Graphite Cast Iron · Multiscale model · Constitutive model

\section{Introduction}

The automotive industry is increasingly facing major challenges related to higher standards decreasing the pollution caused by the emission of greenhouse gases, and the resulting need for lighter structures. Optimal design implies having efficient tools to accurately predict the life of structures under any load and especially fatigue loadings. One possible strategy is based on the fundamental assumption that it is possible to decouple the cyclic mechanical response of the material from fatigue damage [12]. It is usually referred to as a locally coupled approach [6]. The

\author{
L. Augustins \\ PSA Peugeot Citroen \\ Route de Gisy, 78943 Velizy-Villacoublay Cedex, France \\ E-mail: louis.augustins@mpsa.com \\ R. Billardon \\ Messier-Bugatti-Dowty \\ Inovel Parc Sud, 78140 Vélizy-Villacoublay, France \\ E-mail: rene.billardon@safranmbd.com \\ F. Hild \\ LMT Cachan, ENS Cachan/CNRS/University Paris Saclay \\ 61 avenue du président Wilson, 94235 Cachan cedex, France \\ E-mail: hild@lmt.ens-cachan.fr
}


latter requires a number of assumptions to be checked, which are mainly related to the stabilization of the local mechanical variable histories under cyclic loadings.

This work focuses on the thermomechanical fatigue behavior of automotive brake disks. One of the critical points of the design process, as part of the previously described approach, is the correct description of the anisothermal thermomechanical behavior of the material, namely, flake graphite cast iron (FGCI) in most applications. The thermomechanical properties of FGCI depend on the quality and the distribution of flakes and on the matrix composition. Although graphite flakes, with a length ranging from 0.03 to $1 \mathrm{~mm}$, have very little influence on the compressive strength of the matrix, they considerably reduce its tensile strength. Owing to the very low strength of graphite, the flakes behave like cracks [11]. This phenomenon induces a notch sensitivity, causing FGCI to become more brittle than spheroidal graphite cast irons [25]. When macrocracks initiate, they propagate almost instantaneously. In FGCIs, flakes can be seen as microcracks leading to tensile failure (without large elongations or necking) following the graphite flake distribution. Flake debonding under tensile loadings is one of the causes of the nonlinear stress-strain response for relatively low stress values [16], which induces a strong asymmetry of the tension/compression behavior.

Several multiscale models of FGCIs, which are based on finite element simulations, have been devised $[30,13,17]$ to study the influence of the microstructure, in particular the size of flakes, on the mechanical response. The authors were able to simulate cyclic tests with good agreements. The major drawback of these models, which postulate an initially damaged condition, is that they do not allow the simulation of a progressive debonding of graphite flakes; possibly, the modeling of this phenomenon is very complex and cannot be easily parameterized.

Further, many models, either specific or not to FGCIs $[26,14,29,19,20,2,8]$, account for a dissymmetry between the behavior in tension and in compression through the modification of the yield surface, by introducing stress invariants, such as the hydrostatic pressure, in the formulation. The most commonly used model is that proposed by Hejlm [19], which is implemented in the commercial finite element code ABAQus. These models make it possible to represent the overall dissymmetry but fail to account for either the loss of stiffness related to flake debonding, or the stiffness recovery. As an illustration of this lack of precision, the simulation of a symmetric strain controlled cyclic test was achieved using a two-kinematic hardening elastoplastic model with a Drucker-Prager yield surface. Results of this simulation are shown in Figure 1, where the limits of such models are exhibited.

In this paper, after a brief presentation of the experimental database, other multiscale models are proposed leading to a precise description of FGCI behavior. An energetic analysis of these models allows their thermodynamic accuracy to be validated and leads to the formulation of a one dimensional rheological model, which is able to describe precisely the material response under cyclic loading histories.

\section{Experimental database}

In order to study the complex behavior of brake discs gray cast iron, a comprehensive experimental campaign was conducted. The material used to make automotive brakes is a pearlitic FGCI whose chemical composition is presented in Table 1. 


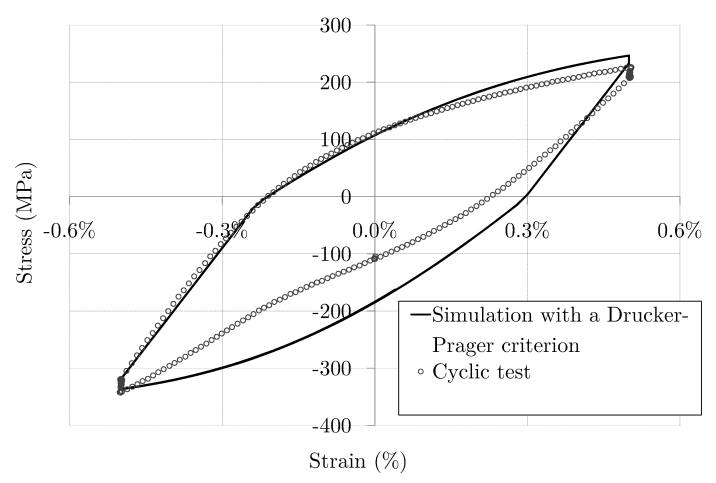

Fig. 1: Simulation of a cyclic test with an elastoplastic model and Drucker-Prager criterion

The flake distribution is uniform, with sizes ranging between 60 and $250 \mu \mathrm{m}$. It is therefore of type IA4-5 [1]. Figure 2 shows a micrograph of the studied grade.

Table 1: Chemical composition (wt.\%) of the studied FGCI

\begin{tabular}{llllllllllll}
\hline $\mathrm{C}$ & $\mathrm{Si}$ & $\mathrm{Mn}$ & $\mathrm{S}$ & $\mathrm{P}$ & $\mathrm{Ni}$ & $\mathrm{Cr}$ & $\mathrm{Mo}$ & $\mathrm{Cu}$ & $\mathrm{Sn}$ & $\mathrm{Ti}$ & $\mathrm{Fe}$ \\
\hline 3.4 & 2.45 & 0.40 & 0.15 & 0.15 & 0.25 & 0.20 & 0.10 & 0.60 & 0.10 & 0.03 & Bal. \\
\hline
\end{tabular}

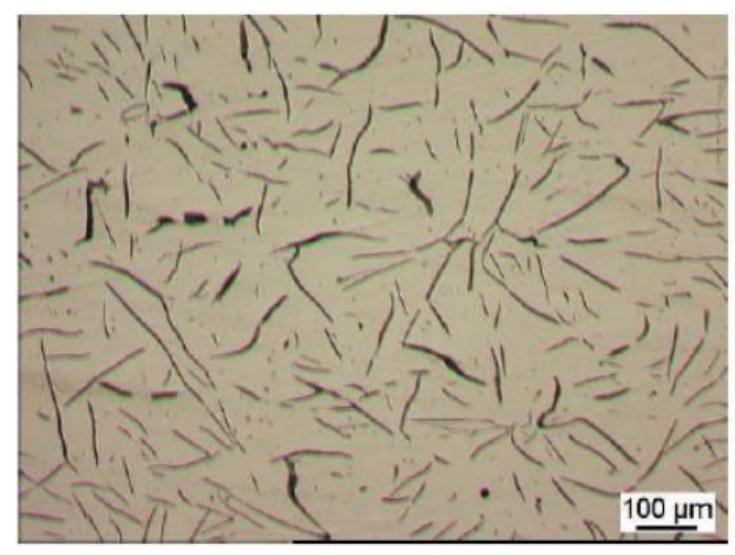

Fig. 2: Micrography of flake graphite cast iron

The experimental database includes monotonic tensile and compressive tests (Figure 3), cyclic tests with dwell phases in tension and compression (Figure 4). All the tests were performed from $20^{\circ} \mathrm{C}$ up to $600^{\circ} \mathrm{C}$ on cylindrical samples, which 
are machined from the ring of a brake disk, in order to be representative of actual components. For the monotonic tests shown in Figure 3, Young's moduli in tension and compression are the same, but a nonlinear stress-strain relationship appears in tension for low stress values (i.e. less than $100 \mathrm{MPa}$ ). Furthermore, stress levels achieved in compression are much higher than those achieved in tension, for symmetric strain levels.

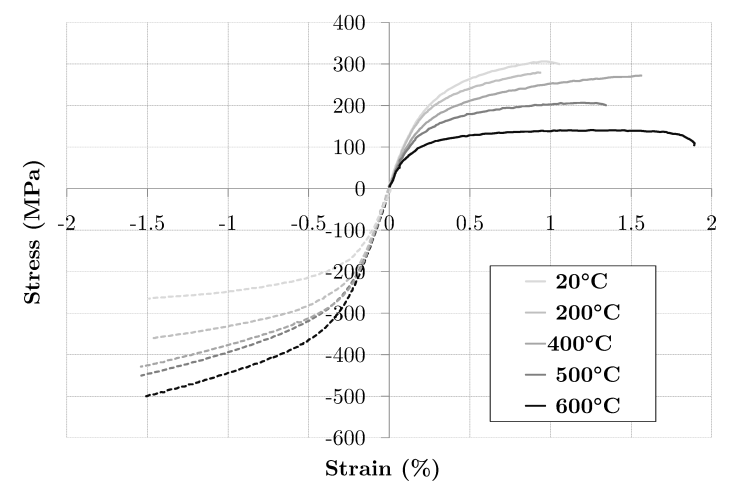

Fig. 3: Monotonic tests performed at different temperatures for the considered FGCI

Cyclic tests consist of a succession of cycles performed at a given strain level $\varepsilon_{1}$. This level varies during the test and its initial value is $\varepsilon_{1}=0.1 \%$. A cycle is composed of:

- a compressive phase from $\varepsilon=0$ to $\varepsilon=-\varepsilon_{1}$ of 2.5 -s duration;

- a dwell phase in compression when $\varepsilon=-\varepsilon_{1}$ of a temperature related duration;

- a compressive/tensile phase when $\varepsilon=-\varepsilon_{1}$ to $\varepsilon=\varepsilon_{1}$ of 5 -s duration;

- a dwell phase in tension when $\varepsilon=\varepsilon_{1}$ of a temperature related duration;

- an unloading phase back to zero strain of 2.5-s duration.

After six cycles at this strain level $\varepsilon_{1}$ (leading to plastic shakedown), the next strain level is considered. During the test, the following values are successively reached for $\varepsilon_{1}: 0.1 \%, 0.2 \%, 0.3 \%, 0.4 \%$ and $0.5 \%$. A strong dissymmetry is observed between tensile and compressive phases (Figure 4), not only in terms of maximum stresses but also in terms of observed elastic modulus. Another particularity is the form of the hysteresis loop, which is characterized by the presence of an inflection point.

Some observations, which are summarized in Figure 5, are derived from the study of the experimental results:

- a strong dissymmetry exists in terms of maximum stress level between tension and compression;

- during loading phases, the current Young's modulus is identical to the initial modulus;

- Young's moduli during the unloading phases are lower than the initial modulus;

- during unloading phases, there is an inflection point corresponding to the gradual closure of cracks induced by matrix/graphite debonding during tensile phases, which gives rise to an increased macroscopic stiffness. 


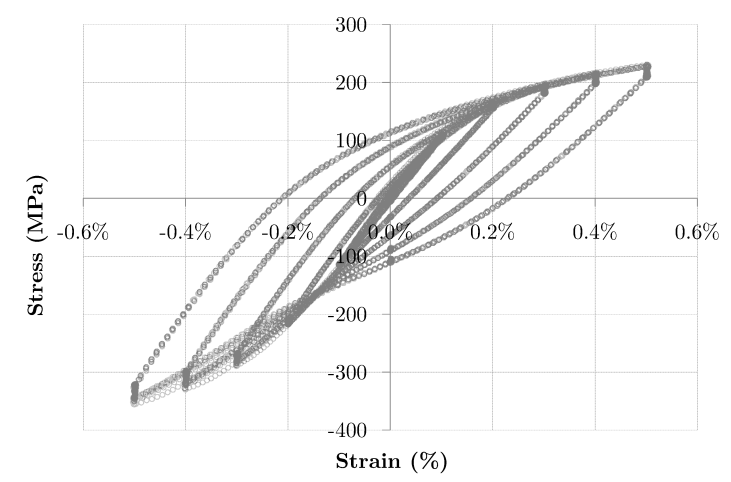

Fig. 4: Cyclic test at room temperature on the considered FGCI

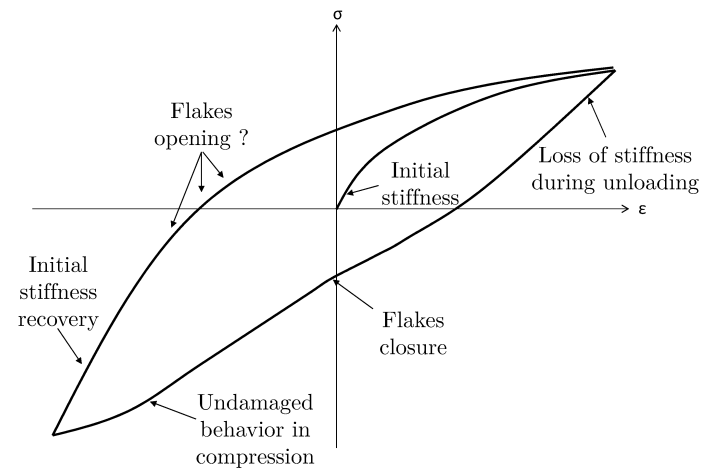

Fig. 5: Schematic behavior of FGCI

\section{Multiscale models}

The detailed understanding of strain and damage mechanisms in FGCI is the key step in the development of a constitutive model. As mentioned in the introduction, multiscale approaches offer a number of answers but fail to simulate accurately the behavior of the material. In this section, unit cells representative of the material are introduced to quantify accurately the phenomena of debonding, opening and closure of the graphite flakes. All the computations are conducted with a general finite element solver (i.e. ABAQus) that uses an implicit integration scheme.

\subsection{Constitutive model of the matrix}

With the experimental database, a constitutive model for the matrix is defined. Cyclic tests show that there is no cyclic softening nor hardening. This result suggests that hardening is purely kinematic (i.e. no isotropic component). Monotonic compressive tests exhibit a highly nonlinear portion in the stress-strain relationship, which is followed by a linear response up to $2 \%$ strain (Figure 6 ). The choice 
Table 2: Matrix parameters $-20^{\circ} \mathrm{C}$

\begin{tabular}{llllll}
\hline $\mathrm{E}(\mathrm{GPa})$ & $\nu$ & $\sigma_{y}$ & $C_{1}(\mathrm{MPa})$ & $\gamma_{1}$ & $C_{2}(\mathrm{MPa})$ \\
\hline 119 & 0,28 & 181 & 230000 & 1634 & 17300 \\
\hline
\end{tabular}

is made to use two kinematic hardening variables [10], one nonlinear $\boldsymbol{X}_{\mathbf{1}}$ [4] and another one $\boldsymbol{X}_{\mathbf{2}}$, which is linear [28]. Since this section only deals with simulations of the behavior at room temperature, an elastoplastic law (i.e. no viscoplasticity) is considered. The representation of the behavior at higher temperatures is addressed in Section 4. Table 2 presents the parameters identified at $20^{\circ} \mathrm{C}$. The simulation shown in Figure 6 describes very well the experimental data and validates the choice of a two-kinematic hardening model.

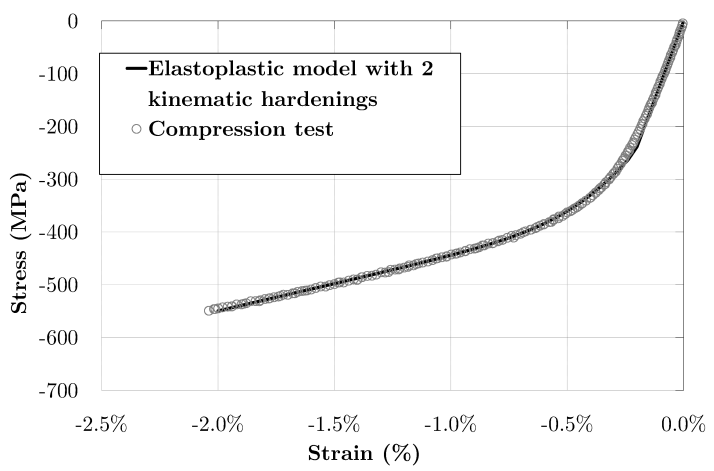

Fig. 6: Simulation of the compression test with the identified elastoplastic model

\subsection{Axisymmetric crack model}

The first model is inspired by that proposed by Russel [30] but displays significant differences, notably in terms of the description of flake debonding. An axisymmetric cell having a flat central crack is defined thereby allowing the three-dimensional effects of stress concentrations at the crack tip to be accurately represented. This crack is an idealization of the graphite flakes perpendicular to the loading direction. The crack surfaces are in contact accounting for closure during unloading phases. The crack propagation is controlled by the applied stress or strain through the use of the $*$ Debond option in ABAQUS, which allows the release of initially linked nodes. Thus, the progressive debonding of the graphite flakes, depending upon the applied load, is only parameterized by the crack size.

The boundary conditions applied to the cell are (Figure 7):

- the upper horizontal face remains horizontal during loading: $y_{H F}=y_{p 1}$;

- the lateral vertical face remains vertical during loading $x_{L F}=x_{p 2}$. 


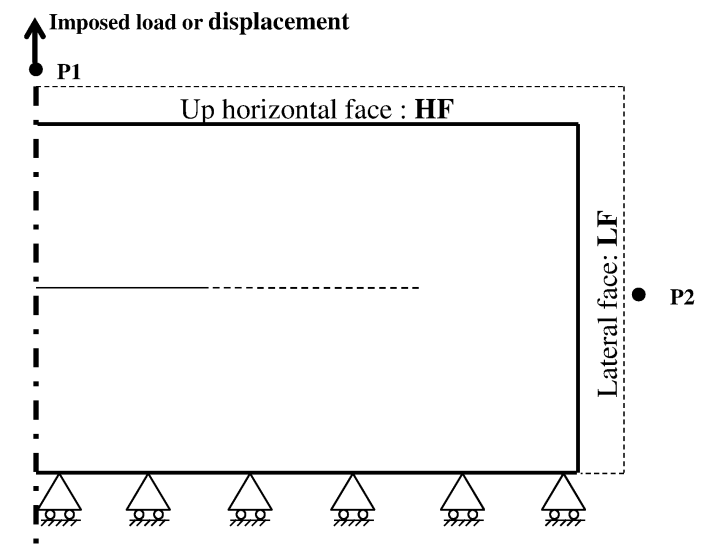

Fig. 7: Axisymmetric crack model

The cell mesh is fine enough to simulate accurately crack opening, namely, elements near the crack tip are $50 \mu \mathrm{m}$ in size for a maximum cell radius of 7 $\mathrm{mm}$. An attempt is then made to identify the crack growth law as a function of the applied stress, in order to obtain a simulated stress-strain curve as close as possible to the stress-strain curve recorded during the monotonic tensile test. The crack growth law is determined "manually" in this exploratory phase, namely, a 20 point tabular evolution of the crack length $a$ as a function of the applied stress is determined step by step from the monotonic tension test. The principle is to fit the experimental tensile test with the appropriate crack size evolution. It is then easy to simulate the cyclic test, incrementing the crack size to its corresponding value, using the previously defined crack opening law, when switching to the new strain level amplitude. The simulation of the cyclic test begins with a strain range of $0.2 \%$ and a pre-existing crack. The response of the model is shown in Figure 8.

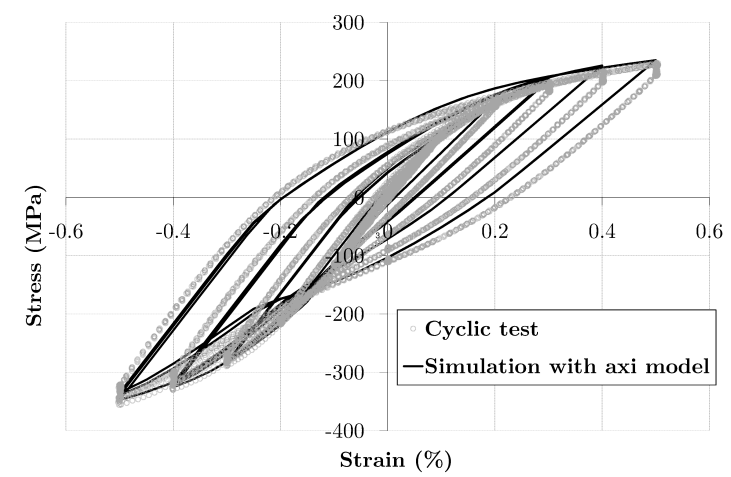

Fig. 8: Comparison of the axisymmetric crack model and the cyclic test 
It is found that the correlation between the model and the experimental data is very good not only during loading phases but also during unloading (i.e. elastic) phases, with a reduced stiffness very close to the measured one. Because the constitutive law is only elastoplastic, the dwell phases with stress relaxation are not simulated. The inflection point that corresponds to crack closure is represented accurately. However, the model predicts that this point, which is experimentally stationary, shifts slightly in the area of negative strains for each increment of the strain amplitude. One hypothesis is to consider that this point is experimentally stationary owing to a wide variety both in size and distribution of the flakes, allowing a gradual closure. Another hypothesis is to consider that the single crack model induces significant stress concentrations at the crack tip, most likely higher than those present in the actual material. The tensile stress concentration generates compressive residual stresses and a large area of positive plastic strains, which then "delay" the complete closure of the crack.

Damage identification The numerical model allows the concept of damage to be addressed. The crack is related to a damage variable $D$ through a decrease of the effective section. The effective stress $\tilde{\sigma}$ introduced by Kachanov [21] is defined as

$$
\tilde{\sigma}=\frac{\sigma}{(1-D)}
$$

The damage variable then reads

$$
D=1-\frac{\tilde{E}}{E}
$$

where $\tilde{E}$ is the current Young's modulus, calculated from an elastic simulation.

Knowing the crack size $a$ as a function of the applied stress $\sigma$, it is possible to directly link $D$ to $\sigma$. The change of $D$ with the applied stress is thus obtained as shown in Figure 9. This result indicates that the flake debonding only occurs after a threshold value and then follows a logarithmic evolution. The yield stress in compression is about $180 \mathrm{MPa}$, which means that damage in tension occurs during the elastic regime.

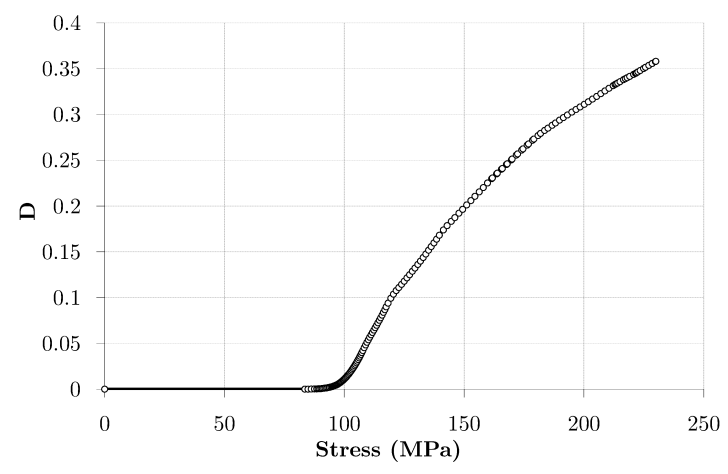

Fig. 9: Damage growth law 


\subsection{Five-bar model}

Along the same lines as the elastoplastic bar model of Downing [13], and given the knowledge of the damage growth law as a function of the applied load, a rheological model made of five bars (i.e. two-dimensional plane stress elements) with different sections is considered to represent the cyclic test shown in Section 2, to the exclusion of any other test. Figure 10 schematically shows the way the fivebar system is working. Bar number 1 with the largest section accounts for the uncracked ligament whose vertical displacements are forbidden on its lower side. Its section $S_{1}$ is directly linked to the maximum damage level corresponding to the strain level of $0.5 \%$, denoted by $D_{0.5} \%$. The section of bar 1 is defined as

$$
S_{1}=S_{T}\left(1-D_{0.5 \%}\right)
$$

where $S_{T}$ is the total section of the $k$ bars, $S_{T}=\sum_{k} S_{k}$. The other four bars correspond to the cracked area. They are in contact with a rigid support, and each bar corresponds to a discretization of damage, which is linked to a strain level. The contact can be open or fully bonded. For a strain level of $0.5 \%$, the contact is open for all bars except bar number 1. For a strain level of $0.4 \%$, the contact is open for bar number 5, 4 and 3 and closed elsewhere. Section $S_{2}$ of bar 2 reads

$$
S_{2}=S_{T}\left(1-D_{0.4} \%\right)-S_{1}
$$

For a strain level of $0.3 \%$, the contact is open for bars number 5 and 4 and closed elsewhere. Section $S_{3}$ of bar 3 becomes

$$
S_{3}=S_{T}\left(1-D_{0.3 \%}\right)-S_{1}-S_{2}
$$

For a strain level of $0.2 \%$, the contact is open for bar number 5 and closed elsewhere so that section $S_{4}$ reads

$$
S_{4}=S_{T}\left(1-D_{0.2 \%}\right)-S_{1}-S_{2}-S_{3}
$$

Last, the section of bar number 5 reads

$$
S_{5}=S_{T}-S_{1}-S_{2}-S_{3}-S_{4}
$$

Displacements of the upper faces of all the bars are kinematically connected to a single master node that can be driven in a load or displacement controlled mode. During the simulation of the cyclic test at $20^{\circ} \mathrm{C}$, the contact condition of the cracked bars 2, 3, 4 and 5 changes from bonded to open, depending on the simulated strain level. The displacement of the master node, as in the test, is "strain" controlled.

The simulations of the rheological model are shown in Figure 11. The predictions are very close to both the experimental data and the results obtained with the axysimmetric crack model. There is still a slight shift of the inflection point toward negative strains at each strain level increment, but the amplitude of the shift is much smaller. This shift can be explained by the absence of effects related to the high stress concentrations in the vicinity of the crack tips, a pattern that is more representative of the actual microstructure characterized by a high density of flakes isotropically distributed. 


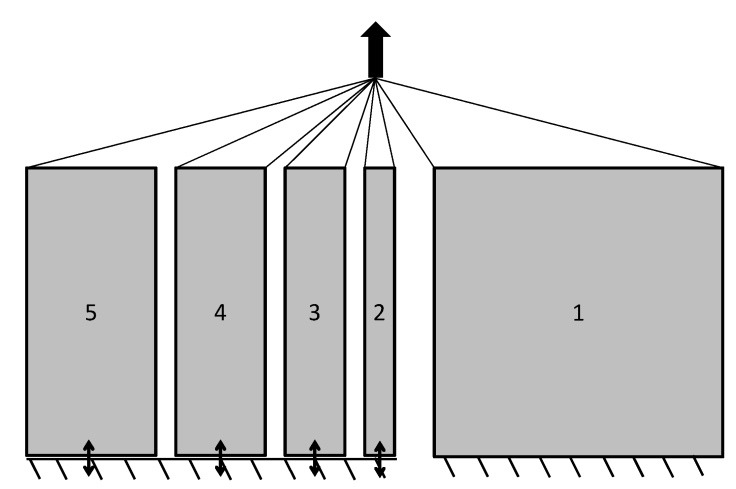

Fig. 10: Five-bar rheological model

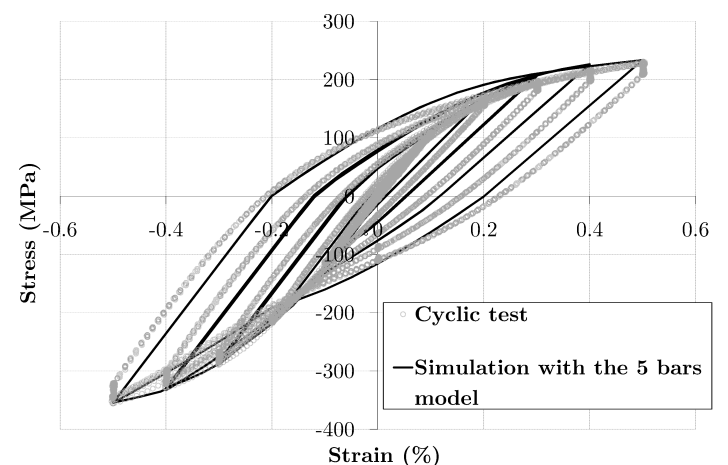

Fig. 11: Prediction of the 5-bar model and experimental data of the cyclic test at $20^{\circ} \mathrm{C}$

When the contact condition for a bar changes from bonded to open, it means that the stress in the considered bar is equal to zero, as well as its elastic strain; only an inelastic strain appeared during compressive phase remains, which means that the bars will keep a constant length during the "open" phase. The consequence is that the open bars (i.e. the open flakes) will close for a global strain level equal to that reached at the time of opening.

\subsection{Energy balance analysis}

In order to compare the two previous models from an energetic point of view, an energy balance analysis is performed on both models.

\section{Thermodynamic framework}

The thermodynamic framework defined below is that used for the undamaged matrix representation. For an elastoplastic model with one nonlinear and 
one linear kinematic hardening, state variables are $\varepsilon, \varepsilon_{p}$ and $\boldsymbol{\alpha}$ associated with the thermodynamic forces $\boldsymbol{\sigma}, \boldsymbol{\Lambda}_{\boldsymbol{\varepsilon}_{p}}$ and $\boldsymbol{X}$.

Von Mises' yield surface is written as

$$
f(\boldsymbol{\sigma}, \boldsymbol{X})=(\boldsymbol{\sigma}-\boldsymbol{X})_{e q}-\sigma_{0}
$$

where $\boldsymbol{X}=\boldsymbol{X}_{1}+\boldsymbol{X}_{2}$, and $\sigma_{0}$ the initial yield stress. The free energy is chosen as the state potential [18]

$$
\rho \omega=\frac{1}{2}\left(\varepsilon-\varepsilon_{\boldsymbol{p}}\right): \mathbb{C}:\left(\varepsilon-\varepsilon_{\boldsymbol{p}}\right)+\omega_{p}\left(\boldsymbol{\alpha}_{\mathbf{1}}, \boldsymbol{\alpha}_{\mathbf{2}}\right)
$$

where $\rho$ is the mass density, $\omega$ the specific free energy, $\mathbb{C}$ Hooke's tensor and $\omega_{p}\left(\boldsymbol{\alpha}_{\boldsymbol{i}}\right)=\frac{1}{3} \sum_{i} C_{i} \boldsymbol{\alpha}_{\boldsymbol{i}}: \boldsymbol{\alpha}_{\boldsymbol{i}},(i=1,2)$ the energy stored related to the hardenings.

The state laws become

$$
\boldsymbol{\sigma}=\rho \frac{\partial \omega}{\partial \varepsilon} ; \boldsymbol{\Lambda}_{\varepsilon_{p}}=-\rho \frac{\partial \omega}{\partial \varepsilon_{p}}=\boldsymbol{\sigma} ; \boldsymbol{X}_{\boldsymbol{i}}=-\rho \frac{\partial \omega}{\partial \boldsymbol{\alpha}_{\boldsymbol{i}}}, i=1,2
$$

The dissipation potential $F$, is different from the yield criterion $f$ (i.e. non-associated plasticity [22])

$$
F(\boldsymbol{\sigma}, \boldsymbol{X})=(\boldsymbol{\sigma}-\boldsymbol{X})_{e q}-\sigma_{0}+\frac{3 \gamma}{4 C} \mathbf{X}_{1}: \boldsymbol{X}_{1}
$$

where $C$ is the hardening modulus, and $\gamma$ the inverse of a characteristic strain associated with the nonlinearity of the hardening. The growth laws read

$$
\begin{gathered}
\dot{\varepsilon}_{p}=\dot{\lambda} \frac{\partial F}{\partial \boldsymbol{\sigma}}=\dot{\lambda} \frac{3}{2} \frac{(\boldsymbol{s}-\boldsymbol{X})}{(\boldsymbol{\sigma}-\boldsymbol{X})_{e q}} \\
\dot{\boldsymbol{\alpha}}_{1}=\dot{\lambda} \frac{\partial F}{\partial \boldsymbol{X}_{1}}=-\dot{\boldsymbol{\varepsilon}}_{p}+\dot{\lambda} \frac{3 \gamma}{2 C_{1}} \boldsymbol{X}_{1} ; \dot{\boldsymbol{\alpha}}_{2}=\dot{\lambda} \frac{\partial F}{\partial \boldsymbol{X}_{2}}=-\dot{\boldsymbol{\varepsilon}}_{p}
\end{gathered}
$$

The cumulated plastic strain rate $\dot{p}$ is deduced from energy equivalence

$$
(\boldsymbol{\sigma}-\boldsymbol{X})_{e q} \dot{p}=(\boldsymbol{\sigma}-\boldsymbol{X}): \dot{\varepsilon}^{p}
$$

and corresponds to the plasticity multiplier

$$
\dot{p}=\dot{\lambda}=\sqrt{\frac{2}{3} \dot{\boldsymbol{\varepsilon}}^{p}: \dot{\boldsymbol{\varepsilon}}^{p}}
$$

The intrinsic dissipation is then expressed as

$$
\mathcal{D}_{i}=\boldsymbol{\sigma}: \dot{\varepsilon}-\rho \frac{\partial \omega}{\partial \mathcal{A}_{i}}: \dot{\mathcal{A}}_{i}
$$

where $\mathcal{A}_{i}$ are all the internal variables except the plastic strain tensor so that

$$
\mathcal{D}_{i}=\left(\sigma_{0}+\frac{3 \gamma}{2 C} \boldsymbol{X}_{1}: \boldsymbol{X}_{1}\right) \dot{p}
$$

Under isothermal conditions, the supplied mechanical work is decomposed into

- the free energy: $\rho \omega=\omega_{e}+\omega_{s}$, itself made of:

- the recoverable elastic energy: $\omega_{e}=\frac{1}{2} \varepsilon_{e}: \mathbb{C}: \varepsilon_{e}$

- and the stored energy: $\omega_{s}(\boldsymbol{\alpha})=\frac{1}{3}\left(C_{1} \boldsymbol{\alpha}_{1}: \boldsymbol{\alpha}_{1}+C_{2} \boldsymbol{\alpha}_{2}: \boldsymbol{\alpha}_{2}\right)$

- and the dissipated energy $D_{1}$, which is obtained by integrating $\left(\sigma_{0}+\frac{3 \gamma}{2 C_{1}} \boldsymbol{X}_{1}: \boldsymbol{X}_{1}\right) \dot{p}$ over the load history. 
Energetic calculations

The analysis of energy balance is carried out on a tensile loading. When a cyclic test is simulated on the axisymmetric crack model, ABAQUs straightforwardly delivers a series of data:

- the so-called plastic work $\psi_{p}$ through ELPD output variable [31], which gives access to the total plastic work, integrated over the whole model,

- the total strain energy $\omega_{e T}$ through ELSE output variable [31], which is also integrated over the whole model.

Consequently, other data have to be determined:

- the stored energy $\psi_{s}$, including the part stored by hardening $\omega_{h}$ and the energy related to the residual stresses $\omega_{s}$.

- The dissipated energy $D_{1}$.

The energy $\omega_{h}$ is obtained from an ABAQUS UVARM user subroutine allowing to integrate over the entire model the energy stored by the hardenings

$$
\omega_{h}=2 \pi \sum_{i=1}^{n}\left[\left(\frac{3}{4 C_{1}} \boldsymbol{X}_{1}^{i}: \boldsymbol{X}_{1}^{i}+\frac{3}{4 C_{2}} \boldsymbol{X}_{2}^{i}: \boldsymbol{X}_{2}^{i}\right) r_{i} S_{i}\right]
$$

where $S_{i}$ is the area of an element, $r_{i}$ the radius of its centroid, and $n$ the total number of elements in the model. The dissipated energy $D_{1}$ is obtained by subtracting $\omega_{h}$ from the plastic work $\psi_{p}: D_{1}=\psi_{p}-\omega_{h}$.

To determine the stored energy associated with the presence of residual stresses $\omega_{s}$, an elastic unloading must be performed at each increment [3]; the stored energy (i.e. residual part of $\omega_{e T}$ ) is that associated with the residual stresses. The recoverable elastic energy $\omega_{e}$ is then equal to $\omega_{e T}-\omega_{s}$. The free energy $\rho \omega$ is finally equal to $\psi_{s}+\omega_{e}$.

Working along similar lines, it is possible to conduct an energetic analysis of the 5-bar model. A Finite Element calculation is carried out for each level of damage (i.e. for strains ranging from $0.2 \%$ to $0.5 \%$ ). The energy stored in hardenings is then calculated as

$$
\omega_{h}=\sum_{i=1}^{n}\left[\left(\frac{3}{4 C_{1}} \boldsymbol{X}_{1}^{i}: \boldsymbol{X}_{1}^{i}+\frac{3}{4 C_{2}} \boldsymbol{X}_{2}^{i}: \boldsymbol{X}_{2}^{i}\right) S_{i}\right]
$$

It is worth noting that there is no energy related to residual stresses in this model. Since the different bars are independent of each other and the stress field in each bar is homogeneous, no residual stresses are generated in the case of a tensile loading followed by an elastic unloading. For the axisymmetric crack model, residual stresses are related to the plastic zone surrounding the crack tip.

The energy balance, per unit area, is shown in Figure 12. An equivalence between the two models is observed, thus allowing a constitutive model based on the 5-bar model to be developed. 


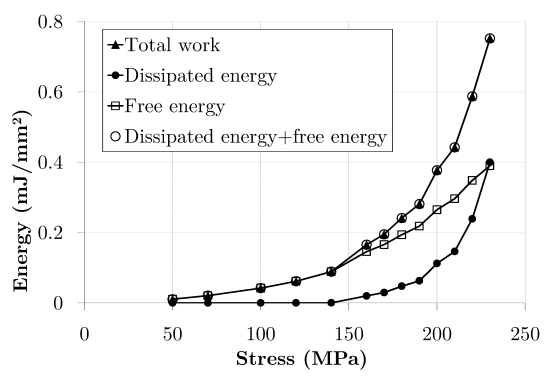

(a) Axisymmetric crack model

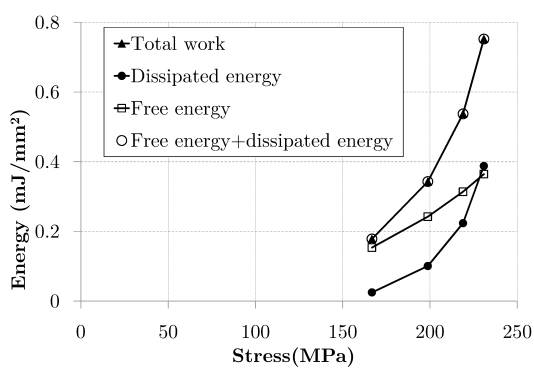

(b) 5-bar model

Fig. 12: Various energetic contributions in a tensile test when two different models are considered

\section{One dimensional rheological model}

\subsection{Theory}

The 5-bar model introduced in Section 3.3 helped us understand the strain mechanisms of FGCI as well as the flake graphite opening and closure conditions from a macroscopic point of view. Based on this model, a one-dimensional two-branch rheological model displayed in Figure 13 is proposed in this section.

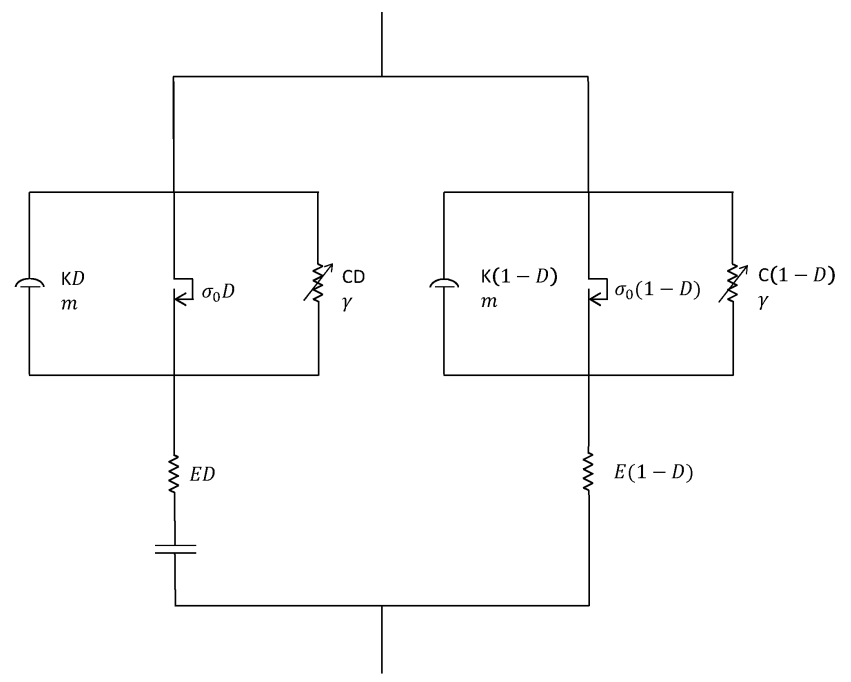

Fig. 13: Rheological model consisting of two branches: the left part is related to the flakes and the right part defines the matrix

The model consists of one branch that describes the matrix, i.e. the undamaged part of the material (the right branch, called the matrix branch) and a second 
branch corresponds to the areas surrounding the debonded graphite flakes (the left branch, called the crack branch), such that the latter, depending on the loading conditions may or may not transmit a load. When the flakes are open, the crack branch must remain inactive and the entire load is carried by the matrix branch. Conversely, when the flakes are closed, the load is carried by both branches.

The case considered hereafter is that of a non-constrained medium, with a preexisting crack, whose length corresponds to a specific level of damage $D$. During a tensile loading, applying a weight $(1-D)$ to several coefficients of the constitutive model is sufficient to bring about the damaged material behavior. During a compressive loading, the behavior is that of the undamaged material since the crack is closed. A weight $D$ is then applied to the same coefficients in the crack branch in order to equate the sum of the stresses in the two branches and the stress of the undamaged material.

Under cyclic loadings, the flakes opening and closure conditions need to be defined. They are schematically shown in Figure 13 by the element with two horizontal bars on the crack branch. The deactivation condition is the same as that described in Section 3.2, namely, the crack closes at the same strain level as that for which it had opened. The opening condition remains to be defined. In the present rheological model, this condition is quite simple. No tensile stresses can be carried by the crack branch. The opening (and therefore deactivation) condition of the crack branch corresponds to the instant when the stress becomes positive. During this period of deactivation, the state variables do not change and the elastic strain is equal to zero.

In order to demonstrate which coefficients should be multiplied by $D$ and $(1-D)$, the same case is studied, namely, that of an unconstrained medium with a pre-existing crack submitted to a monotonic compressive loading. The overall stress is equal to the sum of the stresses in the crack branch, denoted by $\sigma_{c}$, and that of the matrix branch, denoted by $\sigma_{m}$. In the elastic case, the Young's modulus is affected by damage and the overall stress reads

$$
\sigma=\sigma_{c}+\sigma_{m}=E D \varepsilon+E(1-D) \varepsilon=E \varepsilon
$$

In the viscous-elastic case, only Norton's law $K$ parameter is affected, thereby leading to the overall viscous stress

$$
\sigma_{v}=\sigma_{v c}+\sigma_{v m}=K D \varepsilon_{v}^{1 / m}+K(1-D) \varepsilon_{v}^{1 / m}=K \varepsilon_{v}^{1 / m}
$$

Last, for the elastoplastic case with nonlinear kinematic hardening, only the hardening modulus $C$ is influenced by damage, leading to the overall plastic stress

$$
\sigma=\sigma_{c}+\sigma_{m}=\frac{C D}{\gamma}\left(1-e^{-\gamma \varepsilon_{p}}\right)+\frac{C(1-D)}{\gamma}\left(1-e^{-\gamma \varepsilon_{p}}\right)=\frac{C}{\gamma}\left(1-e^{-\gamma \varepsilon_{p}}\right)
$$

The thermodynamic framework chosen to describe in one dimension the undamaged material is defined below. This framework is that of generalized standard materials [15], with the unified elastoviscoplastic model proposed by Chaboche [9], which takes into account viscous effects present at high temperatures. The state potential is the free energy density

$$
\rho \omega=\frac{1}{2}\left(\varepsilon-\varepsilon_{t h}-\varepsilon_{v p}\right) E\left(\varepsilon-\varepsilon_{t h}-\varepsilon_{v p}\right)+\sum_{i} \frac{1}{2} C_{i} \alpha_{i}^{2}
$$


where $\varepsilon_{t h}$ is the thermal strain and $\varepsilon_{v p}$ the viscoplastic strain. The state laws are defined as

$$
\sigma=\rho \frac{\partial \omega}{\partial \varepsilon}=E \varepsilon_{e} ; X_{i}=-\rho \frac{\partial \omega}{\partial \alpha_{i}}=-C_{i} \alpha_{i}
$$

The first kinematic hardening $X_{1}$ is assumed to be nonlinear, the second, $X_{2}$, being linear. The function $F$ is here defined by

$$
F=\sigma-\sum_{i} X_{i}-\sigma_{0}+\frac{\gamma X_{1}^{2}}{2 C_{1}}=f+\frac{\gamma X_{1}^{2}}{2 C_{1}}
$$

In viscoplasticity, the viscoplastic multiplier $\dot{\lambda}$ is obtained via Norton's law $\dot{\lambda}=\left\langle\frac{f}{K}\right\rangle^{m}$. The growth laws are determined by applying the generalized normality rule

$$
\dot{\varepsilon}_{v p}=\dot{\lambda} \frac{\partial F}{\partial \sigma}=\left\langle\frac{f}{K}\right\rangle^{m} ; \dot{\alpha_{1}}=\dot{\lambda} \frac{\partial F}{\partial X_{1}}=-\dot{\varepsilon}_{v p}(1+\gamma) ; \dot{\alpha_{2}}=\dot{\lambda} \frac{\partial F}{\partial X_{2}}=-\dot{\varepsilon}_{v p}
$$

Finally, the cumulative viscoplastic strain rate is deduced from the energy equivalence (Equation (9))

$$
\dot{p}=\dot{\lambda}=\left\langle\frac{f}{K}\right\rangle^{m}=\dot{\varepsilon}_{v p}
$$

\subsection{Numerical integration of the constitutive equations}

The 1D two-branch constitutive model introduced in Section 4 was implemented using an implicit integration scheme [27] in ZEBuLoN [32]. The implicit scheme is a generalized middle point $\theta$-method [23]. It consists of assessing the rate of change of variables $v_{i}$ at time $t+\Delta t$ such that

$$
\dot{v}_{i}(t+\theta \Delta t)=\frac{v_{i}(t+\Delta t)-v_{i}(t)}{\Delta t}=\frac{\Delta v_{i}}{\Delta t}
$$

The quantities $\dot{v}_{i}$ are estimated from the growth laws with physical quantities expressed at time $t+\theta \Delta t$. This scheme is a generalization of the explicit scheme (i.e. $\theta=0$ ) and the fully implicit Euler method (i.e. $\theta=1$ ). In the present case, choosing $\theta=1$ is sufficient. The solution of the numerical problem in structural mechanics consists of calculating the stress increment $\Delta \boldsymbol{\sigma}$ induced by a total strain increment $\Delta \varepsilon$ prescribed during $\Delta t$.

Hereafter and unless explicitly stated, variables $v_{i}$ are always expressed at time $t+\theta \Delta t$, all parameters of the material being measured at temperature $T(t+$ $\theta \Delta t$ ) and $\Delta v_{i}$ is the increment of $v_{i}$ between times $t$ and $t+\Delta t$. The first order approximation $v_{i} \equiv v_{i}(t+\theta \Delta t)=v_{i}(t)+\theta \Delta v_{i}$ is used, where $v_{i}(t)$ is assumed to be known. To further simplify the notations, in what follows the integration scheme is described on the undamaged material. 
Residual vector

Newton's method numerically minimizes the local residual defined as

$$
\{R\}=\left\{\Delta v_{i}\right\}-\Delta t\left\{\dot{v}_{i}(t+\theta \Delta t)\right\}
$$

The chosen integration variables are $\left\{\varepsilon_{e}, \boldsymbol{\alpha}_{1}, \boldsymbol{\alpha}_{2}, p\right\}$, so that the residual vector components are expressed as

$$
\begin{gathered}
R_{\varepsilon_{e}}=\Delta \varepsilon_{e}-(\Delta \varepsilon-\Delta p) \\
R_{p}=\Delta p-\Delta t\left\langle\frac{f}{K}\right\rangle^{m} \\
R_{\alpha_{1}}=\Delta \alpha_{1}+\Delta p\left(1+\gamma \alpha_{1}\right) \\
R_{\alpha_{2}}=\Delta \alpha_{2}+\Delta p
\end{gathered}
$$

\section{Jacobian matrix}

The nonlinear problem to be solved is a $4 \times 4$ dimension system. The solution using Newton's method requires knowing the Jacobian matrix $\boldsymbol{J}$ of the system, determined here analytically, and defined by components such that

$$
J_{i j}=\frac{\partial R_{i}}{\partial \Delta v_{j}}
$$

It is written as

$$
\boldsymbol{J}=\left(\begin{array}{llll}
\frac{\partial R_{\varepsilon_{e}}}{\partial \Delta \varepsilon_{e}} & \frac{\partial R_{\varepsilon_{e}}}{\partial \Delta \alpha_{1}} & \frac{\partial R_{\varepsilon_{e}}}{\partial \Delta \alpha_{2}} & \frac{\partial R_{\varepsilon_{e}}}{\partial \Delta \tilde{p}} \\
\frac{\partial R_{\alpha_{1}}}{\partial \Delta \varepsilon_{e}} & \frac{\partial R_{\alpha_{1}}}{\partial \Delta \alpha_{1}} & \frac{\partial R_{\alpha_{1}}}{\partial \Delta \alpha_{2}} & \frac{\partial R_{\alpha_{1}}}{\partial \Delta \tilde{p}} \\
\frac{\partial R_{\alpha_{2}}}{\partial \Delta \varepsilon_{e}} & \frac{\partial R_{\alpha_{2}}}{\partial \Delta \alpha_{1}} & \frac{\partial R_{\alpha_{2}}}{\partial \Delta \alpha_{2}} & \frac{\partial R_{\alpha_{2}}}{\partial \Delta \tilde{p}} \\
\frac{\partial R_{\tilde{p}}}{\partial \Delta \varepsilon_{e}} & \frac{\partial R_{\tilde{p}}}{\partial \Delta \alpha_{1}} & \frac{\partial R_{\tilde{p}}}{\partial \Delta \alpha_{2}} & \frac{\partial R_{\tilde{p}}}{\partial \Delta \tilde{p}}
\end{array}\right)
$$

Stress increment

The stress increment $\Delta \sigma$ is calculated from the elastic strain $\Delta \varepsilon_{e}$ when Newton's method has converged. The variation of the elastic modulus $E$ must also be taken into account. The implicit integration scheme leads to

$$
\Delta \sigma=\dot{\sigma}(t+\theta \Delta t) \Delta t
$$

which is developed as

$$
\begin{aligned}
\Delta \sigma & =\left(\dot{E \varepsilon_{e}}\right) \Delta t \\
& =E \Delta \varepsilon_{e}+\Delta E \varepsilon_{e}
\end{aligned}
$$


Consistent tangent operator

It is useful to define the consistent tangent matrix (here a scalar) related to the integration scheme to optimize the global convergence and avoid too small time increments. This operator is defined by

$$
L=\frac{\partial \Delta \sigma}{\partial \Delta \varepsilon}
$$

It can be shown that [7]

$$
L=E J_{e e}^{*}
$$

where $J_{e e}^{*}$ is defined from the inverse of the Jacobian $\boldsymbol{J}^{-1}$. It is the block that corresponds to the subscripts related to the elastic strain $\varepsilon_{e}$.

\subsection{Specific implementation in ZEBuLoN}

The solution of the two branches of the rheological model is sought in parallel with a unique total strain increment $\Delta \varepsilon_{e}$, and by assigning factors $D$ or $(1-D)$, as previously defined, to the model parameters. The principle of the algorithm, schematically shown in Figure 14, is the following (subscripts $c$ correspond to the crack branch and subscripts $m$ to the matrix branch):

- The crack branch is deactivated if the stress is greater than or equal to zero. This deactivation occurs at a strain level $\varepsilon_{t}$ called transition strain. The crack branch is activated when the total strain reaches $\varepsilon_{t}$.

- When the branch is deactivated, the internal variables do not change and the elastic strain increment and stress increment, respectively denoted by $\Delta \varepsilon_{e c}$ and $\Delta \sigma_{c}$, are equal to zero. The total stress increment $\Delta \sigma$ is then equal to the stress increment in the matrix branch $\Delta \sigma_{m}$.

- When the crack branch is activated, the total stress is the sum of the contributions of the two branches: $\Delta \sigma=\Delta \sigma_{m}+\Delta \sigma_{c}$.

\subsection{Parameter identification}

To identify the parameters of the model, two steps are needed. First, the growth of the damage variable is determined from the monotonic tests. Then, for a set evolution, the other parameters are tuned. In both cases, ZEBULON software with a Levenberg-Marquardt algorithm [24] is used. The identification method thus involves the optimization of a cost function that evaluates, for a given set of parameters, the correlation between the simulation and the experiment. The error is then expressed in terms of stresses.

Damage growth

Using the monotonic compressive test for each temperature, a first set of parameters related to the undamaged material is tuned. The damage growth as a function of the applied stress is then approximated by a second order polynomial whose coefficients become the parameters of a new identification problem. The 


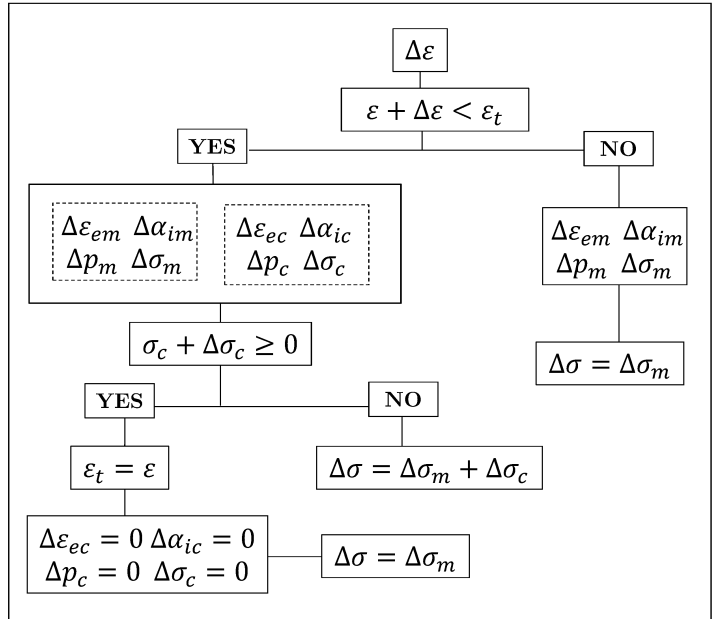

Fig. 14: Algorithmic implementation of the rheological model

latter involves simulating a monotonic tensile test using the previously identified parameters and acting only on the damage variable. The procedure is shown in Figure 15 at room temperature. The damage growth law is easy to use in nonisothermal conditions as it assumes a linear interpolation between two temperatures. For instance, if the damage growth law is defined for $300^{\circ} \mathrm{C}$ and $400^{\circ} \mathrm{C}$, for a given stress, the damage at $350^{\circ} \mathrm{C}$ is a linear interpolation of the damage value at $300^{\circ} \mathrm{C}$ and $400^{\circ} \mathrm{C}$. The resulting polynomial coefficient values as a function of the temperature are summarized in Table 3.

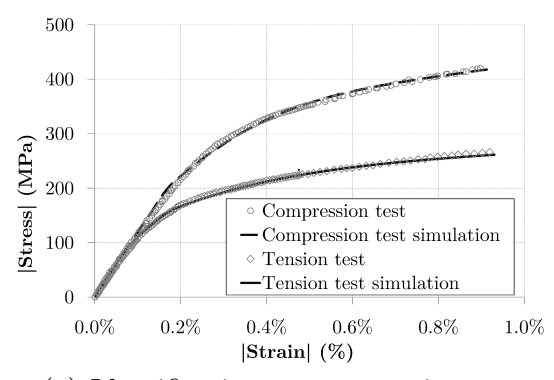

(a) Identification on monotonic tests

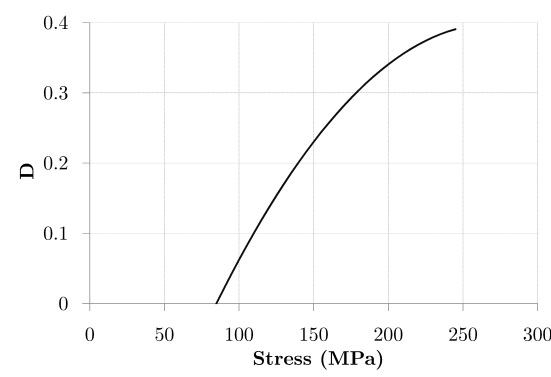

(b) Damage growth law

Fig. 15: Damage identification procedure based upon monotonic tests

\section{Other parameters}

The calibration of a new set of parameters based on cyclic tests is then performed, for which the previously obtained growth law of the damage variable 
Table 3: Polynomial coefficients

\begin{tabular}{llll}
\hline Température $\left({ }^{\circ} \mathrm{C}\right)$ & $a_{0}$ & $a_{1}$ & $a_{2}$ \\
\hline $20^{\circ} \mathrm{C}$ & $-1.15 \mathrm{E}-05$ & $6.23 \mathrm{E}-03$ & $4.46 \mathrm{E}-01$ \\
$200^{\circ} \mathrm{C}$ & $-1.18 \mathrm{E}-05$ & $5.25 \mathrm{E}-03$ & $1.95 \mathrm{E}-01$ \\
$400^{\circ} \mathrm{C}$ & $-1.18 \mathrm{E}-05$ & $5.25 \mathrm{E}-03$ & $1.95 \mathrm{E}-01$ \\
$500^{\circ} \mathrm{C}$ & $-2.20 \mathrm{E}-05$ & $7.55 \mathrm{E}-03$ & $2.70 \mathrm{E}-01$ \\
$600^{\circ} \mathrm{C}$ & $-4.52 \mathrm{E}-05$ & $1.19 \mathrm{E}-02$ & $3.88 \mathrm{E}-01$ \\
$800^{\circ} \mathrm{C}$ & $-1.39 \mathrm{E}-04$ & $2.07 \mathrm{E}-02$ & $3.63 \mathrm{E}-01$ \\
\hline
\end{tabular}

Table 4: Model parameters

\begin{tabular}{llllllll}
\hline Température $\left({ }^{\circ} \mathrm{C}\right)$ & $E(\mathrm{GPa})$ & $\sigma_{y}(\mathrm{MPa})$ & $K$ & $m$ & $C_{1}(M P a)$ & $\gamma$ & $C_{2}(M P a)$ \\
\hline $20^{\circ} \mathrm{C}$ & 119 & 158 & 8.5 & 110 & 151300 & 1010 & 11000 \\
$200^{\circ} \mathrm{C}$ & 119 & 145 & 9.5 & 110 & 127900 & 1140 & 11000 \\
$400^{\circ} \mathrm{C}$ & 110 & 61 & 9 & 306 & 124400 & 1210 & 9000 \\
$500^{\circ} \mathrm{C}$ & 110 & 10 & 6 & 652 & 86000 & 2940 & 8500 \\
$600^{\circ} \mathrm{C}$ & 87 & 1 & 3.7 & 1295 & 10000 & 5000 & 7800 \\
$800^{\circ} \mathrm{C}$ & 62 & 1 & 4.7 & 304 & 500 & 3940 & 100 \\
\hline
\end{tabular}

must be set. This new identification is necessary because the previous one was conducted on monotonic tests with a poor description of the viscous behavior. The dwell phases of the cyclic tests enable for a robust identification of the viscous parameters. Elastic modulus and hardening modulus of the linear hardening $X_{2}$ are identified "manually" on the linear parts of the monotonic tension tests and the five other parameters (Yield stress, two non linear kinematic hardening terms, two viscosity terms) are identified all together on the cyclic test. The same procedure is carried out for the different temperatures. Results are presented in Table 4.

\subsection{Results and discussion}

The simulations of the cyclic tests with the tuned parameters are shown in Figure 16 for four different temperatures. Simulations between $20^{\circ} \mathrm{C}$ and $400^{\circ} \mathrm{C}$ are not presented because the behavior of the considered cast iron is very similar for temperatures up to $300^{\circ} \mathrm{C}$ [Unpublished test report, Exova, 2011].

There is a good description of the behavior under different loading conditions. The loss of stiffness due to tensile loadings and stiffness recovery due to compressive loadings are accurately represented for all the investigated temperatures. The stress relaxation is also very well described, even at high temperatures. For such temperatures (i.e. over $500^{\circ} \mathrm{C}$ ) the description of the inelastic behavior is less accurate than for lower temperatures. This effect is probably due to the use of a relatively simple viscous (i.e. Norton) potential in the constitutive model.

The shift of the inflexion point in the negative strain area is also exacerbated at high temperatures. This phenomenon highlights the limitations of the proposed rheological model and the associated flake opening/closure conditions. 


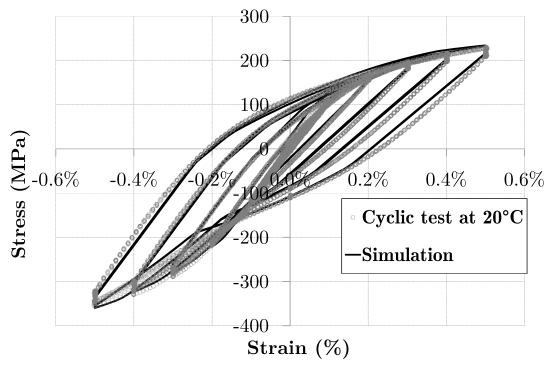

(a) $20^{\circ} \mathrm{C}$

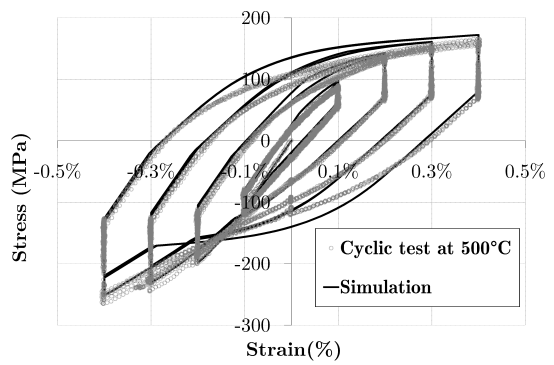

(c) $500^{\circ} \mathrm{C}$

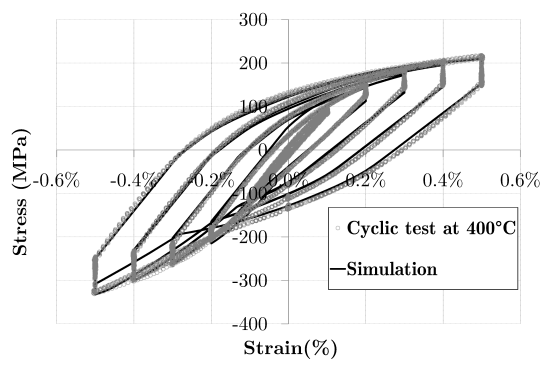

(b) $400^{\circ} \mathrm{C}$

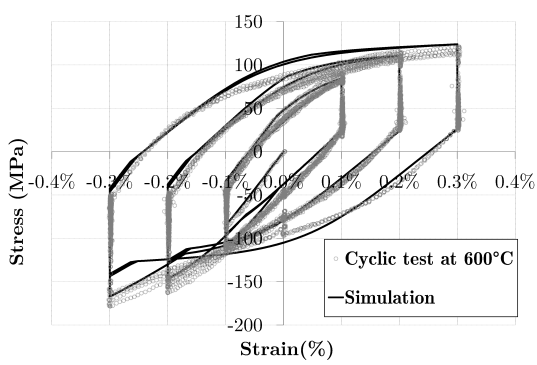

(d) $600^{\circ} \mathrm{C}$

Fig. 16: Comparison of the model predictions and the cyclic tests at four different temperatures

\section{Conclusion}

Owing to the presence of graphite flakes, the behavior of FGCI strongly differs under tensile and compressive loadings. This asymmetry makes the development of a constitutive model very challenging. The simplest approaches based on the modification of the yield surface do not account for complex phenomena involved during the debonding and closure of the flake/matrix interfaces during cyclic loadings.

A rheological model composed of two-dimensional elements with varying sections has helped us define the basis of a constitutive model, which is established for the time being in one dimension, under tensile/compressive loadings. This model accurately represents the principal mechanisms of FGCI. A comparison of this model and the cyclic tests at temperatures ranging from $20^{\circ} \mathrm{C}$ and $600^{\circ} \mathrm{C}$ shows that it is able to describe cyclic loadings at different strain rates, with dwell phases under both tensile and compressive loadings.

It now remains to extend the model proposed to a three-dimensional representation in order to simulate the behavior of brake disks subjected to complex loadings. This issue is addressed in a companion paper [5].

Acknowledgements This work has been performed within the Executive Doctorate in Industry framework, and has been supported by PSA Peugeot Citroën. 


\section{References}

1. AFNOR: Fonderie - fonte à graphite lamellaire. Norme NF EN 1561 (1997)

2. Altenbach, H., Stoychev, G.B., Tushtev, K.N.: On elastoplastic deformation of grey cast iron. Int. J. Past. 17, 719-736 (2001)

3. Aravas, N., Kim, K.S., Leckie, F.A.: On the calculations of the strored energy of cold works. J. Eng. Mat. Tech. 112, 465-470 (1990)

4. Armstrong, P., Frederick, C.: A mathematical representation of the muultiaxial Bauschinger effect. Technical report RD/B/N 731, Central Electricity Generating Board (1966)

5. Augustins, L., Billardon, R., Hild, F.: A constitutive model for flake graphite cast iron automotive brake disks. Induced anisotropic damage model under complex loadings. Continuum Mech. Therm. Submitted article

6. Benallal, A., Billardon, R., Lemaitre, J.: Continuum damage mechanics and local approach to fracture: Numerical procedures. Comp. Meth. Appl. Mech. Eng. 92, 141-155 (1991)

7. Besson, J.: Implementation of material constitutive equations in finite element codes. http://mms2.ensmp.fr/msi_paris/nlfe/transparents/paris_tech2005b.pdf. Accessed: 2013-09-30

8. Brooks, P.C., Barton, D.C., Koetniyom, S.: The development of a material model for cast iron that can be used for brake system analysis. Proceedings of the Inst. Mech. Eng., Part D: J. Automo. Eng. 216(5), 349-362 (2002)

9. Chaboche, J.L.: Description phénoménologique de la viscoplasticité cyclique avec endommagement. Thèse d'Etat, Université Pierre et Marie Curie, Paris 6 (1978)

10. Chaboche, J.L.: Constitutive equations for cyclic plasticity and cyclic viscoplasticity. Int. J. Plast. 5(3), 247-302 (1989)

11. Clough, W.R., Shank, H.E.: The deformation and rupture of grey cast iron. Transactions of the ASM 49, 241-262 (1957)

12. Constantinescu, A., Charkaluk, E., Lederer, G., Verger, L.: A computational approach to thermomechanical fatigue. Int. J. Fatigue 26, 805-818 (2004)

13. Downing, S.D., Socie, D.F.: Stress/strain simulation model for grey cast iron. Int. J. Fatigue 142, 143-148 (1982)

14. Drucker, D., Prager, W.: Soil mechanics and plastic analysis or limit design. Q. Appl. Math 10, 157-165 (1952)

15. Germain, P.: Cours de mécanique des milieux continus. Masson (1973)

16. Gilbert, G.N.J.: An evaluation of the stress-strain properties of flake graphite cast iron in tension and compression. Br. Cast Iron Res. Assoc. J. 7, 745-789 (1959)

17. Haenny, L., Zambelli, G.: The role of the matrix graphite interaction in the tensile behavior of grey cast iron. Eng. Fract. Mech. 19, 113-121 (1984)

18. Halphen, B., Nguyen, Q.S.: Sur les matériaux standards généralisés. Journal de mécanique 14, 39-63 (1975)

19. Hjelm, H.E.: Yield surface for grey cast iron under biaxial stress. Transactions of the Am. Soc. Mech. Eng. 116, 148-154 (1994)

20. Josefson, B.L., Stigh, U., Hjelm, H.E.: A non linear kinematic hardening model for elastoplastic deformations in grey cast iron. J. Eng. Mat. Technol. 117, 145-150 (1995)

21. Kachanov, L.M.: Time of the rupture process under creep conditions. Izv. Akad.Nauk. SSR. Otd Tekh. Nauk. 8, 26-31 (1958)

22. Lemaitre, J., Chaboche, J.L.: Mechanics of Solid Materials. Cambridge University Press, Cambridge (1990)

23. Lemaitre, J., Desmorat, R.: Engineering Damage Mechanics. Springer, New York (2005)

24. Marquardt, D.: An algorithm for least-squares estimation of nonlinear parameters. J. Appl. Math. 11, 431-441 (1963)

25. Meyersberg, H.: Sur la signification du module d'élasticité de la fonte. La Fonte 14, 522 (1934)

26. Mohr, O.: Welche Umstaende bedingen die Elastizitaetsgrenze und den Bruch eines Materials. Zeitschrift des Vereins Deutscher Ingenieure pp. 44-1524 (1900)

27. Ortiz, M., Popov, E.P.: Accuracy and stability of integration algorithms for elastoplastic constitutive relations. Int. J. Num. Meth. Eng. 21, 1561-1576 (1985)

28. Prager, W.: A new method of analyzing stress and strains in work hardening plastic solids. J. Appl. Mech. 23, 493-496 (1956)

29. Raghava, R., Caddell, R.M., Yeh, G.S.Y.: The macroscopic yield behavior of polymers. J. Mat. Sci. 8, 225-232 (1973) 
30. Russell, E.: Finite element simulation of the microstructure of gray cast iron. Fracture Control Program Report No. 33, College of Engineering, University of Illinois (1979)

31. Simulia: Abaqus/Strandard output variable identifiers (section 4.2.1). Abaqus 6.10 Analysis User's Manual (2010)

32. Z-Set: Non-linear material and structure analysis suite. http://www.zset-software.com/. Accessed: 2014-07-27 